\title{
Variations in terminal branches of the popliteal artery: cadaveric study
}

\author{
Łukasz Olewnik $^{1}$ (D) Piotr Łabętowicz ${ }^{3} \cdot$ Michał Podgórski $^{2} \cdot$ Michał Polguj $^{3} \cdot$ Kacper Ruzik $^{1} \cdot$ Mirosław Topol $^{1}$
}

Received: 19 December 2018 / Accepted: 20 May 2019 / Published online: 27 May 2019

(c) The Author(s) 2019

\begin{abstract}
Introduction Identifying the branching pattern of the popliteal artery (PA) is a vital step in planning radiological and surgical procedures involving the anterior and posterior tibial and fibular arteries. The aim of this study was to characterize the course and morphology of the terminal branches of the PA.

Materials and methods The anatomical variations in the branching patterns of the anterior and posterior tibial and fibular arteries were examined in 100 lower limbs fixed in a 10\% formalin solution. A dissection of the popliteal region of the leg was carried out according to a pre-established protocol, using traditional techniques. Morphometric measurements were then obtained twice by two researchers.

Results In most cases (72\%) the PA divides to form the anterior tibial artery (ATA) and a common junction for the posterior tibial and fibular arteries (type I), which further splits into the fibular artery and the posterior tibial artery (PTA). This type was subdivided into two subgroups according to whether the ATA (subgroup a) or the common junction of the posterior tibial and fibular arteries (subgroup b) had the larger diameter. Other identified variations included division of the PA into the ATA and PTA— $8 \%$ (type II), trifurcation-12\% (type III), the division of the PTA into the ATA and FA— $8 \%$ (type IV), and aplasia of the PTA-8\% (type IV).

Conclusion Although the typical PA branching type was observed, it can be classified further into two additional sub-types based on the diameter of the ATA and the common junction of the posterior tibial and fibular arteries.
\end{abstract}

Keywords The popliteal artery $\cdot$ The anterior tibial artery $\cdot$ The fibular artery $\cdot$ The posterior tibial artery $\cdot$ The tibialfibular trunk $\cdot$ New classification

\section{Introduction}

Arterial variations are less common in the lower limbs than the upper limbs $[2,8,13,31,36]$. In addition, the variations that occur in the arterial network of the lower limbs are typically associated with the femoral artery and its main branches [23, 28, 33], with fewer variations being observed for the anterior and posterior tibial arteries and fibular arteries $[5,12,19,26,37]$.

Łukasz Olewnik

lukasz.olewnik@umed.lodz.pl

1 Department of Normal and Clinical Anatomy, Interfaculty Chair of Anatomy and Histology, Medical University of Lodz, Lodz, Poland

2 Department of Diagnostic Imaging Lodz, Polish Mother's Memorial Hospital Research Institute, Lodz, Poland

3 Department of Angiology, Interfaculty Chair of Anatomy and Histology, Medical University of Lodz, Lodz, Poland
The popliteal artery (PA) arises as a continuation of the femoral artery after it passes through the adductor hiatus. The PA runs in the popliteal fossa, and it normally splits at the lower border of the popliteus muscle in the anterior tibial artery (ATA) and the common junction of the posterior tibial and fibular arteries. The ATA passes into the anterior chamber (extensors) of the leg, leaving the posterior chamber (flexors) between the tibia and fibula through the upper opening of the interosseous membrane. The common junction of the posterior tibial and fibular arteries, however, maintains its position in the posterior chamber and splits into the posterior tibial artery (PTA) and the fibular artery (FA). The PA provides blood not only for the knee, including the joint capsule and ligaments, but also for areas of the leg and foot $[4,21]$.

Surgical and radiological procedures of the knee joint are frequent; therefore, knowledge of possible anatomical variants of the PA and its branches is essential for everyday practice. This knowledge is important for orthopaedists 
performing total knee replacements, femoropopliteal and tibial reconstruction surgeries, or distal femur reconstruction and proximal osteotomies [6, 9, 10, 12, 30, 32]. It is also helpful for radiologists performing transluminal stent implantations, angioplasties, embolectomies, or diagnostic angiographies to be familiar with these variants $[12,15]$.

The aim of our study is to determine the variations of terminal branches of the PA that may be relevant for minimizing complications during diagnostic tests and surgical procedures.

\section{Materials and methods}

Anatomical variations of the branching patterns of the ATA, PTA and FA were evaluated in 100 lower limbs (47 female and 53 male). The cadavers were fixed in $10 \%$ formalin solution. The mean age "at death" of the cadavers was 60.7 years (35-85). The cadavers were the property of the Department following donation to the university anatomy program. Each specimen had no signs of surgical intervention in the lower leg region. All lower limbs included in the studies were also free from vascular pathology. The local bioethics commission issued consent for this study.

\section{- Dissection protocol}

The popliteal fossa was approached and popliteal fat was removed. The common fibular nerve, the tibial nerve, and the popliteal vein were identified and separated from the tibial artery. During the dissection, the ATA, the PTA and the FA morphology types were evaluated. Following this, the distance between the intercondylar line and the point of branching of the PA was measured. As intercondylar distance represents a "potential line" between the femoral and tibial condyles, it is a good, palpable topographic site. It also marks the line of the knee joint. If the common junction of the posterior tibial and fibular arteries was present, its length was also measured. In addition, the diameter at the origin site for each artery was measured. The measurements were done with an electronic calliper (Mitutoyo Corporation, Kawasaki-shi, Kanagawa, Japan). Each measurement was accurate to within $0.1 \mathrm{~mm}$, and readings were done twice, by two researchers with high experience in anatomical dissection working independently. The mean value of these two measurements was used for further calculations and analysis.

\section{Statistical analysis}

Statistical analysis was performed using Statistica 12 software (StatSoft Polska, Cracow, Poland). A $p$ value less than 0.05 was considered significant. The results are presented using mean and standard deviation unless otherwise stated.
The Student's $t$ test was used to assess the association between sexes/body sides and types of arterial branching patterns. For continuous variables, normality was first checked with the Shapiro-Wilk test. As the data was not normally distributed, the non-parametric Mann-Whitney test and Wilcoxon signed-rank test were used to compare anthropometric measurements between sexes and body sides, respectively. The Kruskal-Wallis ANOVA test, along with a dedicated post hoc test, was used to compare the measurements taken from different types of the arterial tree.

\section{Results}

Four branching patterns of arteries were identified in the popliteal region:

- Type I (Figs. 1a, 2a). The PA divides into the ATA and the common junction of the posterior tibial and fibular arteries; the latter then splits into the FA and the PTA. This was the most common type, observed in 72 cadavers (72\%). This type was subdivided into sub-type a (in which the ATA had a larger diameter, 42 cases-Fig. 2b) and sub-type $b$ (in which the common junction of the posterior tibial and fibular arteries had a larger diameter, 30 cases-Fig. 2c). In sub-type b, the diameters of the PTA and FA were significantly larger than in sub-type a: $4.00 \pm 1.02$ vs. $3.22 \pm 0.84(p=0.0001)$ for the PTA and $3.80 \pm 1.19$ vs. $2.73 \pm 0.82(p=0.0001)$ for the FA.

- Type II (Figs. 1b, 3a). The PA divides into the ATA and PTA, and then the ATA artery gives rise to the FA. Type II was present in eight cases (8\%).

- Type III (Figs. 1c, 3b). The PA trifurcates into the ATA, PTA, and FA. Type III was visualized in 12 specimens (12\%).

- Type IV (Figs. 1d, 3c). The PA divides into the ATA and FA. The PTA is aplastic. This type was seen in eight specimens $(8 \%)$.

No difference was observed in the distribution of branching patterns between genders $(p=0.2128)$ or body sides $(p=0.4107)$. Morphometric measurements did not differ significantly between body sides, but significant differences were observed between genders (Table 1). With regard to the type of branching pattern, the diameter of the PTA was significantly smaller in type I than in all other types. Additionally, the diameter of the FA was significantly smaller in type I than in type III. Detailed morphometric data regarding the branching patterns are given in Figs. $2 \mathrm{a}-\mathrm{c}$ and $3 \mathrm{a}-\mathrm{c}$. A comparison of the frequency of occurrence of types I, II and III between sexes and body sides is presented in Table 2, while a comparison of morphological parameters with regard to types of branching pattern is presented in Table 3 . 
Fig. 1 Patterns of the terminal branches of the popliteal artery. a Type I of terminal branches of the popliteal artery. Posterior view of the left leg. $P A$ popliteal artery, $A T A$ anterior tibial artery, $T F T$ common junction of the posterior tibial and fibular tibial arteries, PTA posterior tibial artery, $F A$ fibular artery. b Type II of the terminal branches of the popliteal artery. Posterior view of the right leg. $P A$ popliteal artery, ATA anterior tibial artery, $P T A$ posterior tibial artery, $F A$ fibular artery. c Type III of the terminal branches of the popliteal artery. Posterior view of the right leg. $P V$ popliteal vein, $P A$ popliteal artery, ATA anterior tibial artery, $P T A$ posterior tibial artery, $F A$ fibular artery. d Type IV of terminal branches of the popliteal artery—aplasia posterior tibial artery. Posterior view of the left leg. $P A$ popliteal artery, $A T A$ anterior tibial artery, $F A$ fibular artery
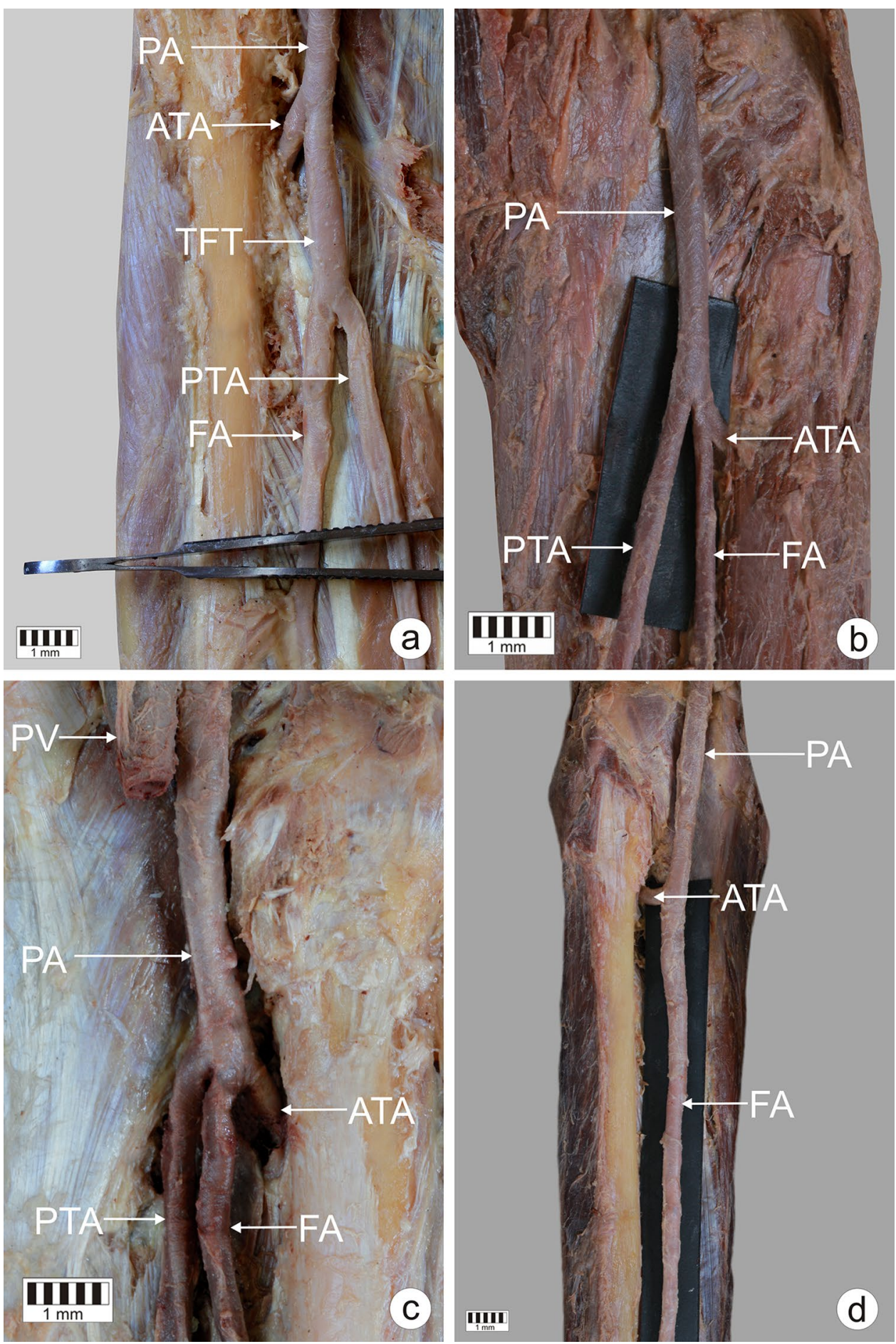

\section{Discussion}

To understand the arterial variations of the terminal branches of the PA, it is necessary to understand the arterial embryology of the lower limb. Most variation results from nonstandard arterial division, aplasia, or hypoplasia. The sciatic artery is a branch of the internal iliac artery and is responsible for supplying blood to the early bundle of the lower limb.
In the 8th week of fetal development, the femoral artery, as a branch of the external iliac artery, connects to the sciatic artery and becomes the main vessel supplying blood to the lower limbs. The sciatic artery regresses proximally and becomes the inferior gluteal artery in adults. The primitive middle and distal segments of the sciatic artery persist to form the definitive popliteal and fibularis arteries. The ATA arises as a branch of the PA, while the PTA is formed by an 
Fig. 2 Schema of type I and its sub-types. Posterior view of the right leg. a Type I of terminal branches of the popliteal artery. $P A$ popliteal artery, ATA anterior tibial artery, $T F T$ common junction of posterior tibial and fibular arteries, PTA posterior tibial artery, $F A$ fibular artery. $\mathbf{b}$ Sub-type A of the type I of the popliteal artery. $P A$ popliteal artery, $A T A$ anterior tibial artery, $T F T$ common junction of posterior tibial and fibular arteries, PTA posterior tibial artery, $F A$ fibular artery. c Sub-type $B$ of the type I of the popliteal artery. PA popliteal artery, ATA anterior tibial artery, TFT common junction of the posterior tibial and fibular arteries, PTA posterior tibial artery, $F A$ fibular artery
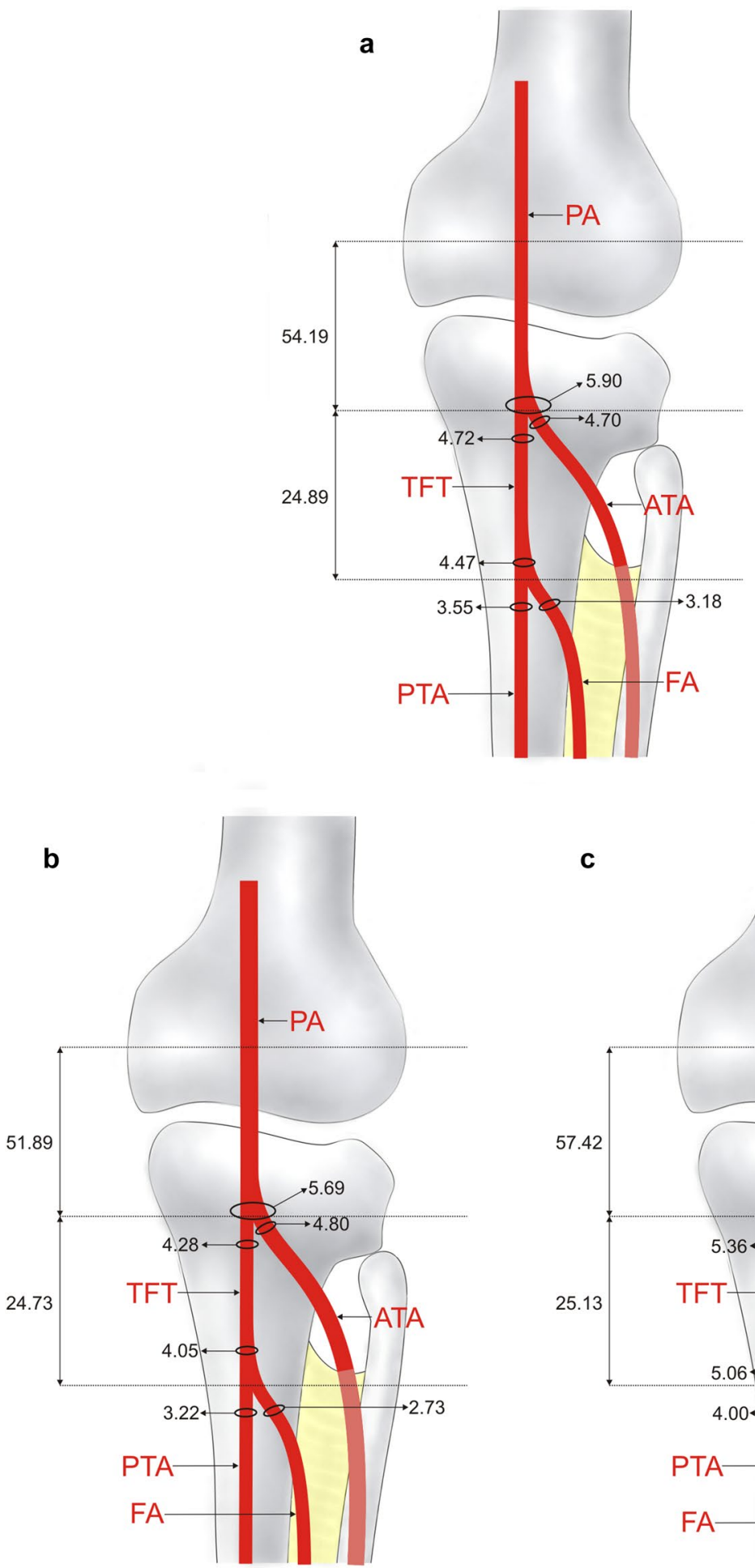

anastomosis between the early distal femoral artery and the PA. Complete vascularisation of the lower limb is established by the end of the 12 th week of pregnancy [19]. The most common morphological variations are associated with high or normal origin, and also concern types $[1,3,5,7,11$, 12, 14, 16-20, 22, 24, 25, 27, 29, 34, 35, 37]. High levels of branching of the PA result from variations in the type of regression of the medial division of the sciatic artery [29]. However, when the anterior sciatic artery bundle fails to regress, a common trunk for the ATA and FA is formed, and when the distal portion of the sciatic artery fails to regress, trifurcation occurs [29]. 

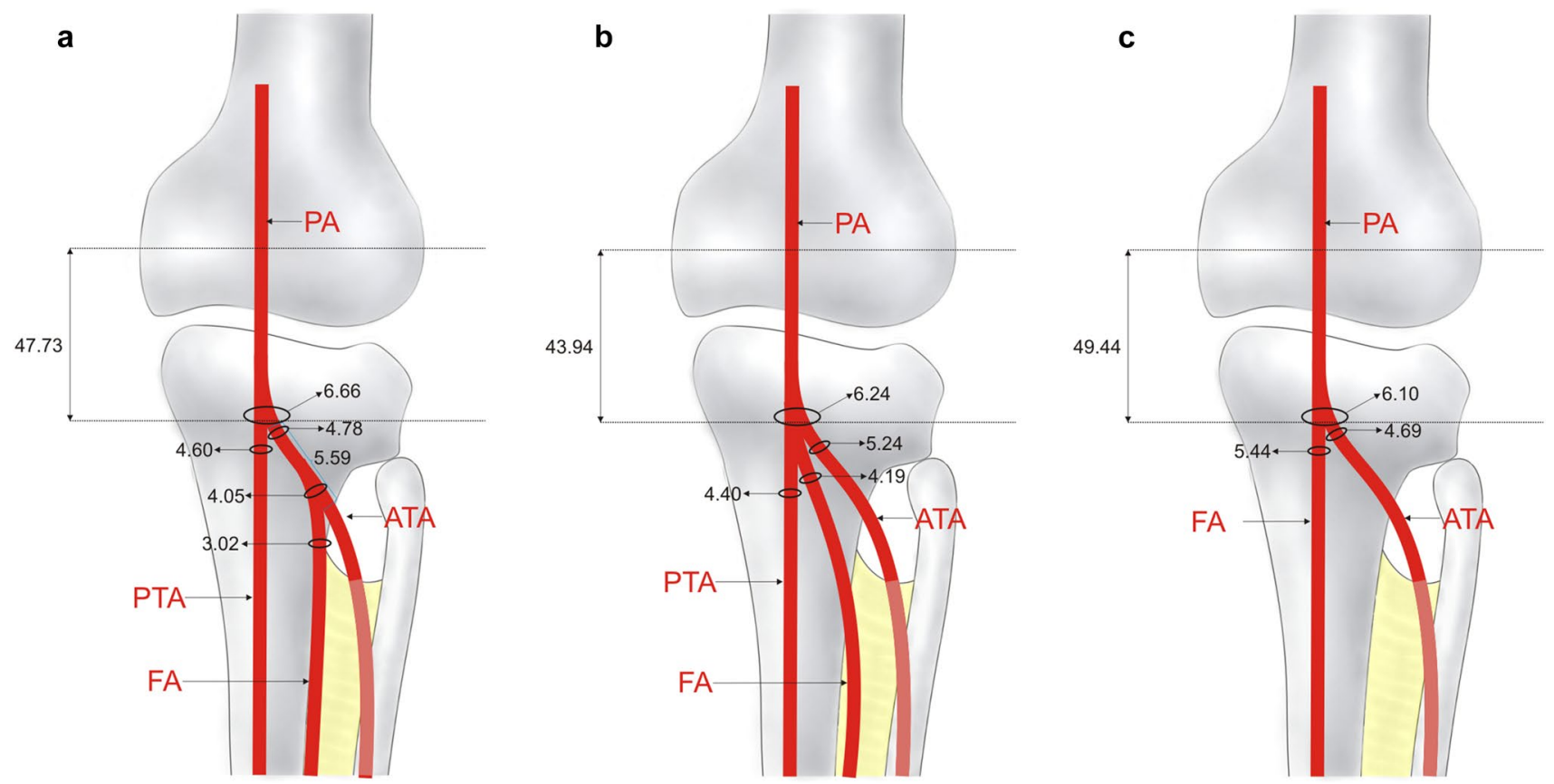

Fig. 3 Schema of type II, III, IV of the terminal branches of the popliteal artery. Posterior view of the right leg. a Type II of the terminal branches of the popliteal artery. PA popliteal artery, ATA anterior tibial artery, PTA posterior tibial artery, FA fibular artery. b Type III of the terminal branches of the popliteal artery. PA popliteal artery, ATA anterior tibial artery, PTA posterior tibial artery, FA fibular artery. c Type IV of terminal branches of the popliteal artery-aplasia posterior tibial artery. $P A$ popliteal artery, $A T A$ anterior tibial artery, $F A$ fibular artery

Table 1 Differences in morphometric measurements between genders [mm]

\begin{tabular}{|c|c|c|c|c|c|c|}
\hline & Females $(n=47)$ & Males $(n=53)$ & $p$ value & Right $(n=54)$ & Left $(n=46)$ & $p$ value \\
\hline Popliteal artery diameter & $5.63(0.85)$ & $6.37(1.18)$ & 0.01 & $6.10(1.05)$ & $5.93(1.16)$ & 0.45 \\
\hline Anterior tibial artery diameter & $4.53(0.83)$ & $4.98(0.98)$ & 0.04 & $4.85(0.97)$ & $4.68(0.90)$ & 0.36 \\
\hline Posterior tibial artery diameter & $3.84(1.08)$ & $3.92(1.11)$ & 0.76 & $3.99(1.20)$ & $3.76(0.95)$ & 0.30 \\
\hline $\begin{array}{l}\text { Common junction of anterior and posterior tibial arteries } \\
\text { with fibular artery origin diameter }\end{array}$ & $4.40(0.73)$ & $4.96(1.12)$ & 0.04 & $4.83(1.10)$ & $4.63(0.92)$ & 0.41 \\
\hline $\begin{array}{l}\text { Common junction of anterior and posterior tibial arteries } \\
\text { with fibular artery end diameter }\end{array}$ & $4.13(0.73)$ & $4.71(1.09)$ & 0.02 & $4.53(1.07)$ & $4.41(0.92)$ & 0.62 \\
\hline $\begin{array}{l}\text { Common junction of anterior and posterior tibial arteries } \\
\text { with fibular artery length }\end{array}$ & $24.23(13.68)$ & $25.37(10.97)$ & 0.55 & $25.42(10.87)$ & $24.37(13.34)$ & 0.72 \\
\hline Fibular artery diameter & $3.00(0.87)$ & $3.54(1.28)$ & 0.05 & $3.47(1.17)$ & $3.10(1.08)$ & 0.12 \\
\hline Intercondylar line-origin of the anterior tibial artery & $49.71(11.60)$ & $54.15(14.57)$ & 0.01 & $53.09(12.08)$ & $50.86(14.80)$ & 0.41 \\
\hline $\begin{array}{l}\text { From the origin of the anterior tibial artery to the origin of } \\
\text { the fibular artery }\end{array}$ & $4.92(0.76)$ & $6.09(3.15)$ & 0.86 & $6.94(3.04)$ & $4.58(1.33)$ & 0.21 \\
\hline $\begin{array}{l}\text { Anterior tibial artery diameter at the origin of the fibular } \\
\text { artery }\end{array}$ & $3.80(0.80)$ & $4.23(1.04)$ & 0.60 & $4.51(0.82)$ & $3.70(0.89)$ & 0.28 \\
\hline
\end{tabular}

Morphological variations of the PA occurred in $28 \%$ of cadavers in this study, which was higher than in other studies, in which the prevalence of variations ranged from 4 to $17.6 \%[1,3,5,7,11,12,14,16-20,22,24,25,27,29,34$, $35,37]$. A few classifications have been proposed for these morphological variants $[16,18]$. A currently used classification by Kim et al. [16], divides the branching pattern of the
PA into three types (I-III), with each type classified further into three sub-types (A-C) (Table 4). Type III is a novelty proposed by Kim et al. [16]; it is characterized by a PA with a normal branching pattern and sequence, but with aplastic or hypoplastic proximal portions of the ATA and/or PTA. The latest systematization of the Kim et al. classification was proposed by Demirtaş et al. [12], who propose the addition 
Table 2 Distribution of branching pattern types between genders and body sides

\begin{tabular}{lllll}
\hline & $\begin{array}{l}\text { Females } \\
(n=47)[n \\
(\%)]\end{array}$ & $\begin{array}{l}\text { Males } \\
(n=53)[n \\
(\%)]\end{array}$ & $\begin{array}{l}\text { Right } \\
(n=54)[n \\
(\%)]\end{array}$ & $\begin{array}{l}\text { Left }(n=46)[n \\
(\%)]\end{array}$ \\
\hline Type I & $30(63.8)$ & $42(79.3)$ & $36(66.7)$ & $36(78.3)$ \\
Type II & $4(8.5)$ & $4(7.6)$ & $4(7.4)$ & $4(8.7)$ \\
Type III & $7(14.9)$ & $5(9.4)$ & $9(16.7)$ & $3(6.5)$ \\
Type IV & $6(12.8)$ & $2(3.8)$ & $5(9.3)$ & $3(6.5)$ \\
$p$ value & 0.25 & & 0.39 & \\
\hline
\end{tabular}

of type ID, characterized by a very long common junction of the posterior tibial and fibular arteries $(110 \mathrm{~mm})$, and type IIID, characterized by a hypoplastic/aplastic PTA. Our present findings indicate the need for the addition of a further subdivision for type IA based on the relative diameters of the ATA and the common junction of the posterior tibial and fibular arteries: more specifically, sub-type A1, characterized by the ATA being wider than the common junction of the posterior tibial and fibular arteries, and sub-type A2, by the common junction of the posterior tibial and fibular arteries being wider than the ATA. Although no type II branching patterns were found in any specimens, further subdivision according to vessel diameter would be advantageous.
The most common variant of the terminal branches of the PA detected by anatomical dissection is type I, with a frequency ranging from 92.5 to $97.3 \%[1,14,18,27,34]$. This has been confirmed by angiographic studies, with the frequency of type I found to range from 88.2 to $95.8 \%$ [3, $7,11,12,15,16,19,20,22,25,35]$. Type I was also found to be the most common variant in the present study, occurring in $72 \%$ of cases (summation of patterns I, II and III). The prevalence of type II branching, according to Kim (high origin), has been found to range from 0.8 to $7.5 \%$ in anatomical studies $[1,14,18,24,27,34]$ and from 1.6 to $7.8 \%$ in angiographic studies $[3,7,11,12,15,16,19,20,22,25$, 35 ]; however, this type was not observed in any of our specimens. Type III (Kim) has only been reported in angiographic studies, with an occurrence ranging from 1 to $7.6 \%[7,11$, $12,15,20,25,35]$. Interestingly, $8 \%$ of the specimens in the present anatomical study displayed type IIIA branching according to Kim (our type IV). More precise differences in the findings of anatomical and angiographic studies are presented in Tables 5 and 6. Interestingly, the present study shows that the diameter of the PTA was significantly smaller in type I than in the other types (II, III), and the diameter of the FA in type I was significantly smaller than in type III; this is an important point for consideration when performing surgical procedures in this area.

Table 3 Comparison of morphological parameters between types of the branching pattern

\begin{tabular}{|c|c|c|c|c|c|}
\hline & Type I $(n=72)$ & Type II $(n=8)$ & Type III $(n=12)$ & Type IV $(n=8)$ & $p$ value \\
\hline Popliteal artery diameter & $5.90(1.15)$ & $6.66(0.97)$ & $6.24(1.06)$ & $6.10(0.59)$ & 0.23 \\
\hline Anterior tibial artery diameter & $4.70(0.92)$ & $4.78(0.74)$ & $5.24(0.84)$ & $4.69(1.30)$ & 0.27 \\
\hline Posterior tibial artery diameter & $3.55(0.99)$ & $4.60(0.61)$ & $4.40(0.75)$ & $5.44(0.81)$ & $<0.01$ \\
\hline $\begin{array}{l}\text { Common junction of anterior and posterior tibial arteries with } \\
\text { fibular artery origin diameter }\end{array}$ & $4.73(1.01)$ & & & & - \\
\hline $\begin{array}{l}\text { Common junction of anterior and posterior tibial arteries with } \\
\text { fibular artery end diameter }\end{array}$ & $4.47(0.99)$ & & & & - \\
\hline $\begin{array}{l}\text { Common junction of anterior and posterior tibial arteries with } \\
\text { fibular artery length }\end{array}$ & $24.90(12.09)$ & & & & - \\
\hline Fibular artery diameter & $3.18(1.12)$ & $3.02(0.51)$ & $4.19(1.22)$ & & 1.00 \\
\hline Intercondylar line-origin of the anterior tibial artery & $54.19(12.21)$ & $47.73(11.59)$ & $43.94(19.27)$ & $49.44(10.60)$ & 0.12 \\
\hline $\begin{array}{l}\text { From the origin of the anterior tibial artery to the origin of the } \\
\text { fibular artery }\end{array}$ & & $5.59(2.36)$ & & & - \\
\hline Anterior tibial artery diameter at the origin of the fibular artery & & $4.05(0.90)$ & & & - \\
\hline
\end{tabular}

Table 4 Classification modified by Kim et al. [16]

\begin{tabular}{llll}
\hline & Type I—normal division & Type II—high division & Type III—aplastic/hypoplastic \\
\hline Sub-type & & & \\
A & Normal pattern & ATA arises at or above popliteus muscle & Hypoplastic-aplastic PTA \\
B & Trifurcation & PTA arises at or above popliteus muscle & Hypoplastic-aplastic ATA \\
C & Anterior tibial-fibular trunk & FA arises at or above popliteus muscle & Hypoplastic-aplastic PTA and ATA \\
\hline
\end{tabular}




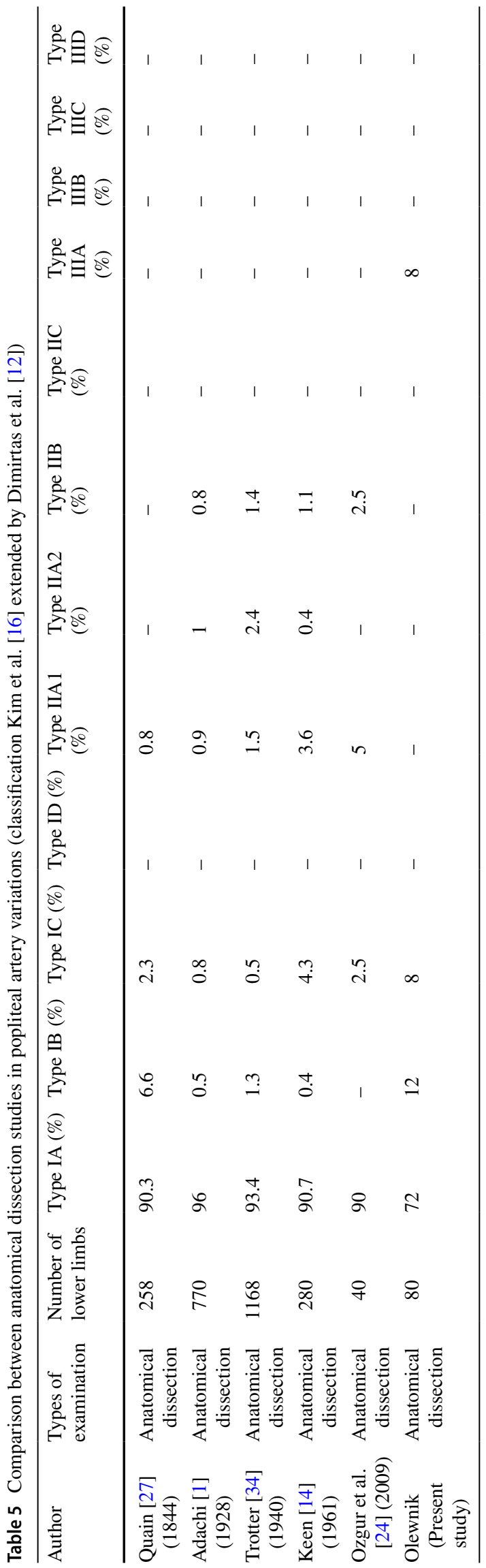

Arterial variations in the terminal branches of the PA have great radiological, orthopedic and surgical importance, as without this precise knowledge, iatrogenic injury is possible during procedures such as vascular graft transplants, embolectomies, surgical repairs or endovascular angioplasties [12, 32]. The presence of highly branched arteries, especially the ATA, and their variability can present a number of arterial complications for such orthopedic interventions as total knee arthroplasties, high tibial osteotomies, posterior cruciate ligament reconstructions, lateral meniscal repairs and arthroscopies [17, 25]. The presence of a type III PA may be particularly problematic in this sense. In the case of a hypoplastic or aplastic PTA, the FA exhibits compensatory hyperplasia and supplies the vascular bed of the PTA with blood. In this case, the FA of the foot gives rise to the lateral plantar artery, but the medial plantar artery is usually absent [37]. The FA is also a significant vessel for the preparation of the fibular graft and gastrocnemius muscle flap [37]. In the case of possible hypoplasia or aplasia of the ATA and/or the PTA, it is particularly important to make a pre-operative determination of the adequacy of the blood supply to the foot after blocking the FA.

The main limitation of our study was the small size of the research sample. Nevertheless, its number is comparable to, or even larger than, those of samples used in similar anatomical studies [24]. The second weakness of the work is that it is based only on anatomical study; its findings would be complemented by data from angiographic studies and would improve the practicality of the prepared classification. Finally, our classification includes an analysis of vessel diameter and an evaluation of dominance. Although we are aware that postmortem changes of vessel walls can influence direct measurements, these changes should be uniform across samples and affect all vessels and are unlikely to demonstrate bias.

\section{Conclusion}

Adequate knowledge of the high morphological variability occurring in the terminal branches of the PA is needed to safely perform correct diagnostic and surgical procedures in this region of the body. PTA diameter was significantly smaller in type I than in the other types (II, III), and FA diameter in was significantly smaller in type I than type III. Any comprehensive classification of PA branching pattern should, therefore, include two sub-types based on vessel diameter for type IA and all versions of type II. 


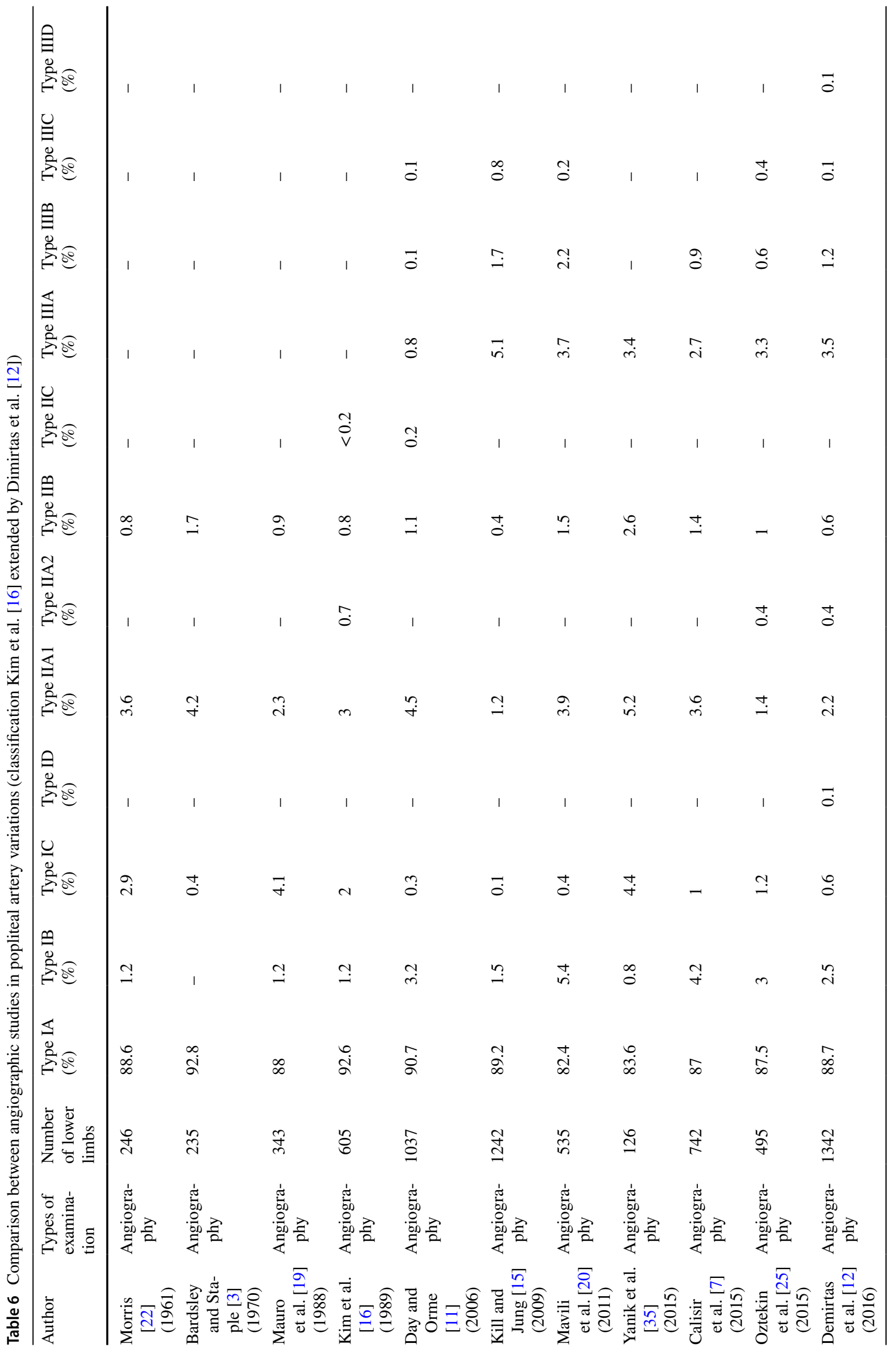


Acknowledgements The authors wish to express their gratitude to all those who donated their bodies to medical science.

Author contributions ŁO (D.P.T., Ph.D.) -Assistant-project development, data collection and management, data analysis and manuscript writing. PŁ (M.D., Ph.D.)—Assistant-data collection, data analysis, manuscript editing. MP (M.D., Ph.D.)—Assistant—data collection, data analysis, manuscript editing. MP (M.D., Ph.D.)—Professordata analysis, manuscript editing. KR-student-data collection. MT (M.D., Ph.D.) Professor-manuscript editing. All authors have read and approved the manuscript.

Funding The authors have no financial or personal relationship with any third party whose interests could be positively or negatively influenced by the article's content. This research did not receive any specific grant from funding agencies in the public, commercial, or not-for-profit sectors.

\section{Compliance with ethical standards}

Conflict of interest The authors declare that they have no competing interests.

Ethical approval and consent to participate The anatomical protocol of the study was accepted by Bioethics Committee of Medical University of Lodz (resolution RNN/297/17/KE). The cadavers belong to the Department of Normal and Clinical Anatomy of the Medical University of Lodz. The ultrasonographic protocol of the study was accepted by the Bioethics Committee of Polish Mother's Memorial Hospital Research Institute (resolution 54/2018).

Consent to publish Not applicable.

Availability of data and materials Please contact authors for data requests (Łukasz Olewnik Ph.D.—email address: lukasz.olewnik@ umed.lodz.pl).

Open Access This article is distributed under the terms of the Creative Commons Attribution 4.0 International License (http://creativeco mmons.org/licenses/by/4.0/), which permits unrestricted use, distribution, and reproduction in any medium, provided you give appropriate credit to the original author(s) and the source, provide a link to the Creative Commons license, and indicate if changes were made.

\section{References}

1. Adachi B (1928) Das Arteriensystem der Japaner, vol 2. Verlag der Kais Univ zu Kyoto, Kyoto, pp 18-71

2. Aragão JA, da Silva ACF, Anunciação CB, Reis FP (2017) Median artery of the forearm in human fetuses in northeastern Brazil: anatomical study and review of the literature. Anat Sci Int 92:107111. https://doi.org/10.1007/s12565-015-0322-x

3. Bardsley JL, Staple TW (1970) Variations in branching of the popliteal artery. Radiology 94:581-587. https://doi. org/10.1148/94.3.581

4. Bergman RA, Afifi AK, Miyauchi R (2015) Anatomy atlases: illustrated encyclopedia of human anatomic variation-anatomical variation, radiology anatomy, anatomy atlas. http://www.anato myatlases.org/AnatomicVariants/AnatomyHP.shtml. Accessed 6 Feb 2017
5. Bettaiah A, Venkat S, Saraswathi G (2016) A study of variations in the branching pattern of popliteal artery and its clinical perspective. Int J Res Med Sci 4:3584-3589. https://doi.org/10.18203 /2320-6012.ijrms20162333

6. Binkley F, Edwin J (1958) Surgical approaches to the popliteal artery. Am J Surg 96(2)213-220

7. Calisir C, Simsek S, Tepe M (2015) Variations in the popliteal artery branching in 342 patients studied with peripheral CT angiography using 64-MDCT. Jpn J Radiol 33:13-20. https://doi. org/10.1007/s11604-014-0373-2

8. Chi Z, Yang P, Song D, Li Z, Tang L, Gao W, Song Y, Chu T (2017) Reconstruction of totally degloved fingers: a novel application of the bilobed spiraled innervated radial artery superficial palmar branch perforator flap design provides for primary donor-site closure. Surg Radiol Anat 39:547-557. https://doi.org/10.1007/ s00276-016-1760-4

9. Colborn GL, Lumsden AB, Taylor BS, Skandalakis JE (1994) The surgical anatomy of the popliteal artery. Am Surg 60:238-246

10. Davies B, Husami T, Lewis J, Retrum E (1989) Developmental variations of the popliteal artery and its branches: a clinical correlation. Contemp Surg 34:28-32

11. Day CP, Orme R (2006) Popliteal artery branching patternsan angiographic study. Clin Radiol 61:696-699. https://doi. org/10.1016/j.crad.2006.03.014

12. Demirtaş H, Değirmenci B, Çelik AO, Umul A, Kara M, Aktaş AR, Parpar T (2016) Anatomic variations of popliteal artery: evaluation with 128-section CT-angiography in 1261 lower limbs. Diagn Interv Imaging 97:635-642. https://doi.org/10.1016/j. diii.2016.02.014

13. Haładaj R, Wysiadecki G, Dudkiewicz Z, Polguj M, Topol M (2018) The high origin of the radial artery (brachioradial artery): its anatomical variations, clinical significance, and contribution to the blood supply of the hand. Biomed Res Int 2018:10-11. https ://doi.org/10.1155/2018/1520929

14. Keen JA (1961) A study of the arterial variations in the limbs, with special reference to symmetry of vascular patterns. Am J Anat 108:245-261. https://doi.org/10.1002/aja.1001080303

15. Kil S-W, Jung G-S (2009) Anatomical variations of the popliteal artery and its tibial branches: analysis in 1242 extremities. Cardiovasc Interv Radiol 32:233-240. https://doi.org/10.1007/s0027 0-008-9460-Z

16. Kim D, Orron DE, Skillman JJ (1989) Surgical significance of popliteal arterial variants. A unified angiographic classification. Ann Surg 210:776-781

17. Klecker RJ, Winalski CS, Aliabadi P, Minas T (2008) The aberrant anterior tibial artery. Am J Sports Med 36:720-727. https:// doi.org/10.1177/0363546507311595

18. Marty AT (1986) Arterial variations in man: classification and frequency. J Am Med Assoc 255:2821. https://doi.org/10.1001/ jama.1986.03370200123049

19. Mauro M, Jaques P, Moore M (1988) The popliteal artery and its branches: embryologic basis of normal and variant anatomy. Am J Roentgenol 150:435-437. https://doi.org/10.2214/ajr.150.2.435

20. Mavili E, Donmez H, Kahriman G, Ozaslamaci A, Ozcan N, Tasdemir K (2010) Popliteal artery branching patterns detected by digital substraction angiography. Diagn Interv Radiol 17:80-83. https://doi.org/10.4261/1305-3825.DIR.3141-09.1

21. Moore KL, Dalley AF (1999) Clinical oriented anatomy, 4th edn. Lippincott Williams \& Wilkins, Philadeplphia

22. Morris GC, Beall AC, Berry WB, Feste J, de Bakey ME (1960) Anatomical studies of the distal popliteal artery and its branches. Surg Forum 10:498-502

23. Nasr AY, Badawoud MH, Al-Hayani AA, Hussein AM (2014) Origin of profunda femoris artery and its circumflex femoral branches: anatomical variations and clinical significance. 
Folia Morphol (Warsz) 73:58-67. https://doi.org/10.5603/ FM.2014.0008

24. Ozgur Z, Ucerler H, Aktan Ikiz ZA (2009) Branching patterns of the popliteal artery and its clinical importance. Surg Radiol Anat 31:357-362. https://doi.org/10.1007/s00276-008-0454-y

25. Oztekin PS, Ergun E, Civgın E, Yigit H, Kosar PN (2015) Variants of the popliteal artery terminal branches as detected by multidetector ct angiography. Open Med (Warsaw, Poland) 10:483-491. https://doi.org/10.1515/med-2015-0056

26. Portilho PO, Leite TFdO, Cardoso R, Pires LAS, Silva JG, Chagas CAA (2016) Clinical features of a rare anatomical variation of the posterior tibial and fibular arteries. J Vasc Bras 15:234-238. https ://doi.org/10.1590/1677-5449.003416

27. Quain R (1844) Anatomy of the arteries of the human body. Taylon \& Walton, London, pp 415-422

28. Rajani SJ, Ravat MK, Rajani JK, Bhedi AN (2015) Cadaveric study of profunda femoris artery with some unique variations. J Clin Diagn Res 9:AC01-3. https://doi.org/10.7860/ jcdr/2015/13913.5876

29. Rohan A, Domagała Z, Abu Faraj S, Korykowska A, Klekowski J, Pospiech N, Woźniak S, Gworys B (2018) Branching patterns of the foetal popliteal artery. Folia Morphol (Warsz). https://doi. org/10.5603/fm.a2018.0052

30. Senior HD (1929) Abnormal branching of the human popliteal artery. Am J Anat 44:111-120. https://doi.org/10.1002/aja.10004 40105

31. Singer G, Marterer R, Till H, Schmidt B (2018) A rare anatomic variation of the superficial palmar branch of the radial artery causing pain. Surg Radiol Anat 40:349-352. https://doi. org/10.1007/s00276-017-1936-6

32. Stewart AH, Baird RN (2001) The prevention and early recognition of arterial complications in total knee replacement: a vascular surgical perspective. Knee 8:265-267

33. Tomaszewski KA, Henry BM, Vikse J, Roy J, Pękala PA, Svensen M, Guay DL, Saganiak K, Walocha JA (2016) The origin of the medial circumflex femoral artery: a meta-analysis and proposal of a new classification system. PeerJ 4:e1726. https://doi. org/10.7717/peerj.1726

34. Trotter M (1940) The level of termination of the popliteal artery in the White and the Negro. Am J Phys Anthropol 27:109-118. https://doi.org/10.1002/ajpa.1330270130

35. Yanik B, Bulbul E, Demirpolat G (2015) Variations of the popliteal artery branching with multidetector CT angiography. Surg Radiol Anat 37:223-230. https://doi.org/10.1007/s0027 6-014-1346-y

36. Zekavica A, Milisavljević M, Erić D, Ćurčić B, Popović S, Vitošević B, Dožić A, Śtimec BV, Manojlović R (2017) Vascular anatomy of the thenar eminence: its relevance to a pedicled or free thenar flap. Folia Morphol (Warsz) 76:232-238. https://doi. org/10.5603/FM.a2016.0077

37. Zheng M, Chen C, Qiu Q, Wu C (2016) Ultrasound in diagnosis of anatomical variation of anterior and posterior tibial arteries. Med Ultrason 18:64-69

Publisher's Note Springer Nature remains neutral with regard to jurisdictional claims in published maps and institutional affiliations. 